\title{
VAC.I7 - Quality control process through monitoring hydrazide-activated protein reactions by LC-SRM/MS
}

Marilza B. Corrêa ${ }^{1 \star}$; Renata C. Bastos ${ }^{1}$; Hilton J. do Nascimento ${ }^{1}$; Maria de Lourdes M. Leal ${ }^{1}$; Ivna A. F. B. da Silveira ${ }^{1}$.

1Fiocruz/Bio-Manguinhos.

Introduction: The reductive amination chemistry is a synthetic approach normally used in the development of conjugate vaccines. In this approach, a high carrier protein reactivity is desirable. This improvement occurs by converting the side carboxylate groups of aspartic and glutamic residues into their respective hydrazides. The measurement of this conversion is an analytical challenge due to sample complexity and low concentrations of analytes. The determination of relative activation rate will allow us to monitorize the reproducibility of the method bringing innovation to process quality control

Objective: The aim of this study is to quantify levels of aspartic and glutamic amino acids in proteins hydrolysates. Such quantification will be performed in those chemically modified proteins by isotopic labeling with aTRAQ ${ }^{\mathrm{T}}$ reagent and (LC-SRM/MS) analysis. These procedures intend to determine the degree of activation of protein.

Methodology: Standard protein (SP) was activated by reaction with carbodiimide and hydrazine at $25^{\circ} \mathrm{C}$, for $240 \mathrm{~min}$. The positive control of degree of activation was executed thru evaluating, by comparison, the activated protein isoelectric point with its correspondent native one (Phast SystemTM GE). In hydrolysis step, Gaba amino acid was added as surrogate standards (100umol/L) in order to monitorize the hydrolysis as well as the labeling efficiency. The hydrolyzate was obtained by the action of $6 \mathrm{~mol} / \mathrm{L} \mathrm{HCl}$, at $110{ }^{\circ} \mathrm{C}$ for $24 \mathrm{~h}$, labeled with aTRAQ ${ }^{\mathrm{TM}}$ reagent, and analyzed on an Agilent HPLC system with mass detection (API 3200 SCIEX). The chromatographic separation was performed using a column in gradient mode (RP-C18, 4.6x150mm, 5um). For quantitative analysis, four samples $(\mathrm{n}=4)$ were analyzed in 5 replicates. Data statistical analysis was performed using GraphPad Prism 5 software.

Results: The insertion of basic hydrazide groups significantly altered the isoelectric point (IP) values from 5.85 (SP) to 8.8 (AP). The analytical data is normal distributed, showing for SP (Glu: $\mu$ 85.36; SD 5.43 - Asp: $\mu$ 48.06; SD 3.39) and for AP (Glu: $\mu$ 69,93, SD 10.5; Asp: $\mu$ 39.25, SD 13.0). The surrogate standard had a $>85 \%$ recovery. The independent t-test for samples was applied to the means, showing significant variation (Asp: p 0.0215 and Glup: 0.022), with a confidence level of $95 \%$.

Conclusion: The IP values indicate a significant change in basicity of the activated protein. The independent t-test showed a significant difference between the means in two unrelated groups. The observed concentrations allowed to determine the relative activation rate, which were estimated in the groups' rate of $18 \%$.

Keywords: Amino acid analysis; mass spectrometry; activated protein 